\title{
Assessment of motor outcomes in multiple sclerosis patients with walking impairment treated with Fampridine using motor evoked potentials
}

\section{DANIELA MARILENA TROFIN}

Universitatea de Medicina si Farmacie Gr T Popa lasi Facultatea de Medicina

DAN TROFIN ( $\nabla$ dr.dantrofin@gmail.com )

Universitatea de Medicina si Farmacie Gr T Popa lasi Facultatea de Medicina https://orcid.org/00000002-1480-3467

\section{BOGDAN IGNAT}

Universitatea de Medicina si Farmacie Gr T Popa Iasi Facultatea de Medicina

\section{ANDREI CIOBICA}

Universitatea de Medicina si Farmacie Gr T Popa lasi Facultatea de Medicina

\section{TEODOR STAMATE}

Universitatea de Medicina si Farmacie Gr T Popa lasi Facultatea de Medicina

WALTHER BILD

Universitatea de Medicina si Farmacie Gr T Popa lasi Facultatea de Medicina

\section{Research article}

Keywords: Transcranial Magnetic Stimulation, multiple sclerosis, walking speed, Fampridine

Posted Date: September 8th, 2019

DOl: https://doi.org/10.21203/rs.2.14103/v1

License: (c) (1) This work is licensed under a Creative Commons Attribution 4.0 International License. Read Full License 


\section{Abstract}

Backgound : the purpose of the study is to consider the utility of Transcranial Magnetic Stimulation (TMS) in patients with Multiple Sclerosis (MS) and walking impairment, treated with Fampridine, as correlated with the 25-Foot-Walk test (T25-FW). It is known that clinical benefits should be identified within two to four weeks after starting the treatment; otherwise, if there is no benefit reported by patients, it should be discontinued.

Methods: 15 MS patients with walking impairment, ranked between 3.5 and 7 on the Expanded Disability Status Scale (EDSS), were investigated by T25-FW and TMS. Evaluations were performed prior to Fampridine $10 \mathrm{mg}$ twice daily administration, 5 and 12 days afterwards, and also 1 and 3 months later. The medium age was 42.8 years and an average duration of the disease was 12.06 years.

Results: we assessed an improvement of walking by 2.1 seconds measured on the T25-FW after the first 12 days in 9 patients, which correlated with a 2 miliseconds improvement of the Central Motor Conduction Time (CMCT). For the other 6 patients there was no visible clinical improvement.

Nevertheless, CMCT dropped by $0.5 \mathrm{~ms}$ and the motor conduction speed by 1 millisecond in 4 of these 6 patients. Fampridine administration was continued in the 4 cases. At the end of the 3 months period, their walking speed measured as on the T25-FW also improved by $2 \mathrm{sec}$.

Conclusions: the amelioration of TMS parameters anticipated the improvement of speed on the T25-FW. In spite of the early false negative clinical response, electrophysiological findings could predict a future clinical improvement if treatment is continued.

\section{Introduction}

Multiple sclerosis (MS) is a chronic neurological disease based on the pathophysiological phenomena of demyelination among the Central Nervous System (CNS), which leads to an accumulation of both physical and cognitive disabilities. Impairment of walking is one of the most burdensome clinical characteristics of MS. Deficits are quantified using the Expanded Disability Status Scale (EDSS).

Neurodegeneration and development of symptoms in MS is based on ion channel dysfunctions (1). There are several studies of $\mathrm{Na}+$ and $\mathrm{K}+$ channels that promote ion channel modulation as a promising therapeutic strategy in MS. Therefore, it is known today about the pathological $\mathrm{Na}+$ channel blockade during the acute inflammatory attack, as well as the contribution of increased $\mathrm{Na}+$ channel expression to the cascade of events leading to neurodegeneration, thus the pharmacological approach of this phenomenon in clinical 
trials. Nonetheless, the demyelination process is based on the physiopathology of the $\mathrm{K}(+)$ channels, modulation of the fast $\mathrm{K}(+)$ conductance, in other words the changes in axonal excitability that shift towards normal values, translate into symptoms amelioration (2).

Fampridine in the dose of ten milligrams twice daily may improve gait speed and endurance, as compared to baclofen, however, the positive results seem to relate to less than half patients (3). Fampridine ameliorates conduction in demyelinated axons by enhancing synaptic transmission as a voltage-dependent potassium channel blocker. Its result of improvement of walking speed seems independent of improvement of disability (4). The walking speed and endurance can be quantified using walking tests measurements, such as the 25-foot walk test (T25FW), which is considered to be an objective and reliable evaluation tool fulfilling both efficacy and tolerability criteria (5-7).

In the vast majority of cases, Fampridine's efficacy has been assessed by the use of the T25FW (8). The most utilized therapeutic indication is improvement of walking disability associated to an EDSS ranked between 4 and 7 (9).

Fampridine seems to be usually well tolerated providing clinical benefit on different impaired gait patterns according to the T25W: paraparetic, hemiparetic or ataxic $(7,10)$. The clinical benefit also influenced the EDSS of these patients by significantly decreasing its value. Nevertheless, even in such prospective studies cases that reveal the positive influence of Fampridine in the clinical response, one must also rely to possible mild-moderate adverse events that can occur in up to half of the respondents, as well as accepting the fact that a gait pattern predictive for the clinical efficacy couldn't be identified so far (10). Assessment of nerve excitability may however, in some cases, contribute to identify patients most likely to respond to Fampridine (11). Brambilla L. et al suggest by a multi-instrumental approach based on clinical evaluation scales correlated with neurophysiological methods (Motor Evoked Potential) and Diffusion Tensor Imaging before and after 14 days of treatment, that the modified neurophysiological and neuroradiological parameters correlate with the clinical and subjective improvement of the motor results, thus proposing electrophysiology and neuroimaging as useful tools for assessment of effectiveness and follow-up of MS patients with walking difficulties (12). 


\section{Material And Methods}

\subsection{Background}

Our pilot study was performed in the Neurology Clinic from The Rehabilitation Hospital in Iași, during February 2015 and December 2016. The included patients had Relapse Remitting Multiple Sclerosis (RRMS) and Secondary Progressive Multiple Sclerosis (SPMS), according to the MacDonalds criteria of 2010. The following criteria were being considered at the inclusion in the study: EDSS, presence of walking impairment as a result of disease evolution as opposed to acute impairment as a result of a relapse, as well as absence of contraindications for performing Transcranial Magnetic Stimulation (TMS), especially any history of epileptic discharges.

The study protocol was approved by the Hospital's Ethics Committee. All patients were properly informed, agreed with their participation in the study and signed a consent form. The included patients were tested clinically, and with electrophysiological methods, as well as being well monitored for any possible adverse events during the treatment.

\subsection{The electrophysiological study}

We investigated 15 patients with MS and walking impairment, with EDSS between 3.5 and 7, average 4.6. 13 patients had RRMS, while the other 2, SPRR. The age was between 30 and 55 years old, with average age of 42.8 years. Eight patients were female, representing 53.33\% and 7 male patients, 46.67\%. None of the female participants were pregnant during the study. Five of the 15 patients had paraparetic motor deficit, 4 patients motor deficit on right side of the body, 3 patients on the left side of the body and 3 patients had tetraparetic deficit. The duration of the illness inside the lot was between 5 and 23 years, with an average of 12.06 years.

The purpose of the study was to investigate a possible correlation between clinical (T25-FW) and paraclinical (TMS) parameters, related to the improvement of walking, after administration of Fampridine. The hypothesis is that early electrophysiological findings can act as predictors the future clinical increase of speed in patients that do experience clinical 
improvements. On the other hand, for patients with no evidence of improvement of walking on the T25-FW after 2 weeks of treatment, but amelioration of the Motor Evoked Potential (MEP) latencies at TMS, further administration of Fampridine might be predicting a possible future clinical amelioration.

TMS was performed using the Magstim Rapid ${ }^{\circledR}$ device. We used the butterfly shaped coil and the round shaped coil. The butterfly shaped coil generates a magnetic field of up to 1.2 Tesla, whereas the round shaped coil generates a specific cone shaped magnetic field, thus leading to a diffuse stimulation. This is a good reason to use this round shaped coil in current clinical practice for a more appropriate stimulation of the cervical and lumbar areas of the spine.

MEP was obtained by placing surface electrodes on the abductor digiti minimi muscle in the upper limb and on the tibialis anterior muscle in the lower limb. For follow-up of the evolution of electrophysiological parameters after Fampridine administration, we measured the motor conduction latency and the Central Motor Conduction Time (CMCT), prior to the drug administration, 5 and 12 days after, as well as 1 and 3 months after continuous administration of Fampridine $10 \mathrm{mg}$ twice daily.

For the central stimulation we used the butterfly shaped coil, stimulating the right and left cerebral hemisphere, collecting data from the abductor digiti minimi muscle. For the lower limbs, the stimulation was similar, but collecting info from the tibialis anterior muscle. For the stimulation of the cervical and lumbar regions, we used the round shaped coil, stimulating at C7 and L5 levels, and collecting data from the same two muscles.

\subsection{The clinical evaluation}

We measured the patients' walking speed using the T25-FW scale, at the mentioned interval of time according to Fampridine's first administration. The distance the patients had to walk was $7.62 \mathrm{~m}$ (25 feet). The rhythm was constant for the whole length; the patients went back through the same distance, with time necessary measured as well. The two-timed values obtained were averaged. The walk was being performed in straight line. 
The patients were asked not to lean against the wall, however, in case of important motor deficit, they were allowed to use a cane.

\subsection{Quality of life}

At the end of all the time periods described according to the Fampridine intake, the patients completed a satisfaction questioner regarding the quality of life (QoL). They were asked to appreciate on a scale from 0 to 5 how they relate to the drug in terms of expectations and everyday activities (Table 1).

\begin{tabular}{|c|}
\hline Satisfaction degree \\
\hline 0 = bad \\
\hline 1 = stationary \\
\hline 2 = slightly better \\
\hline 3 = good \\
\hline 4 = very good \\
\hline 5 = exceptionally \\
\hline
\end{tabular}

Table 1. The QoL evaluation scale the patients related to

\subsection{Analysis of data}

The statistical analysis was performed using SPSS, through appliance of the Friedman test for paired samples. Considering the small lot, non-parametric tests were used, because of unequally-distributed data. The effect of administration of the drug was measured both clinical and electrophysiological reported to the mentioned time intervals.

\section{Results}


At the 12 days endpoint analysis, based on the electrophysiological evaluation, the group divided into responders and non-responders. 9 patients presented early amelioration

of the MEP and CMCT latencies, whereas the other 6 showed no significant improvement. The following analysis, repeated measures ANOVA, was conducted only on the group of the responders $(n=9)$.

\subsection{Quality of life}

Regarding the quality of life, a significant main effect of time was observed (Fig. 1), Wilks' Lambda $=0.033, \mathrm{~F}(3,6)=58, \mathrm{p}<0.001$.

When we examined the pairwise comparisons for statistic differences within groups per daily quality of life, we observed significant differences between quality of life at baseline and quality of life on day 12 ( $\mathrm{p}<0.001)$, between quality of life at baseline and quality of life on day 30 ( $\mathrm{p}<0.001$ ), between quality of life at baseline and quality of life on day 90 ( $p<0.001)$. Also, significant differences were observed between quality of life at day 5 and quality of life on day 12 ( $p<0.001$ ), between quality of life at day 5 and quality of life on day 30 ( $\mathrm{p}<0.001)$, between quality of life at day 5 and quality of life on day 90 ( $p$ $<0.001)$. Furthermore, significant differences were observed between quality of life at day 12 and quality of life on day 30 ( $\mathrm{p}<0.001)$ and between quality of life at day 12 and quality of life on day 90 ( $\mathrm{p}=0.003$ ). The only non-significant differences observed were between quality of life baseline and quality of life at day $5(\mathrm{p}=1)$ and between quality of life at day 30 and quality of life at day $90(p=1)$.

Fig. 1 The effect of fampridine on the level of the quality of life

\subsection{Timed-25-Foot-Walk test}

Analysing the Timed-25-Foot-Walk test results, a significant main effect of time was observed (Fig. 2), Wilks' Lambda $=0.011, \mathrm{~F}(4,5)=112.621, \mathrm{p}<0.001$. 
When examining the pairwise comparisons for statistic differences within groups related to the Timed-25-Foot-Walk test, we observed significant differences between the time obtained by the patients at baseline and the time obtained on day 12 ( $p<0.001$ ), between time obtained at baseline and time obtained on day 30 ( $p<0.001)$, between time obtained at baseline and time obtained on day $90(\mathrm{p}<0.001)$. Also significant differences were observed between time obtained by the patients on the 25 Foot Walk test at day 5 and the time obtained on day 12 ( $\mathrm{p}<0.001)$, between time obtained at day 5 and time obtained on day 30 ( $\mathrm{p}<0.001$ ), between time obtained at day 5 and time obtained on day 90 ( $\mathrm{p}<$ 0.001). Significant differences were also observed between time obtained by the patients on the 25 Foot Walk test at day 12 and time obtained on day $30(\mathrm{p}<0.001)$ and between time obtained at day 12 and time obtained on day $90(\mathrm{p}=0.003)$. In addition, significant differences were also observed between time obtained by the patients on the 25 Foot Walk test on day 30 and time obtained on day 90 ( $p<0.001)$. The only non-significant differences observed were between the Timed-25-Foot-Walk test at baseline and Timed-25-Foot-Walk test at day $5(\mathrm{p}=0.132)$.

Fig. 2 The effect of fampridine on the time obtained by the patients on the 25 Foot Walk test

\subsection{The Central Motor Conduction Time (CMCT) from the right hemisphere}

Regarding the Central Motor Conduction Time (CMCT) from the right hemisphere a non- significant main effect of time was observed (Fig 3), Wilks' Lambda $=0.202, F(4,5)$ $=4.931, \mathrm{p}=0.055$.

However, when we examined the pairwise comparisons, to see if there is a statistical difference within groups dealing with the Central Motor Conduction Time (CMCT) from the right hemisphere, we observed significant differences between the CMCT at baseline and CMCT on day $5(\mathrm{p}=0.035)$, between CMCT at baseline and CMCT on day $12(\mathrm{p}=0.029)$, between CMCT at baseline and CMCT on day $90(\mathrm{p}=0.03)$. Also, significant differences were observed between CMCT at day 30 and CMCT on day $90(p=0.005)$. Non-significant 
differences were observed between any other possible pair of time points where the CMCT was measured.

Fig. 3 The effect of fampridine on the time obtained by the patients on the Central Motor Conduction Time (CMCT) from the right hemisphere

\subsection{The Central Motor Conduction Time (CMCT) from the left hemisphere}

Analysis of CMCT from the left hemisphere revealed a significant main effect of time (Fig. 4), Wilks' Lambda $=0.130, \mathrm{~F}(4,5)=8.387, \mathrm{p}<0.019$.

During these next pairwise comparisons, we observed significant differences between the CMCT at baseline and CMCT on day $12(\mathrm{p}=0.009)$, between CMCT at baseline and CMCT on day 90 ( $\mathrm{p}=0.037)$, between CMCT at day 5 and CMCT on day $12(\mathrm{p}=0.031)$. Also, significant differences were observed between CMCT at day 30 and CMCT on day 90 $(p=0.004)$. Non-significant differences were observed between any other possible pair of time points where the CMCT was measured.

Fig. 4 The effect of fampridine on the time obtained by the patients on the Central Motor Conduction Time (CMCT) from the left hemisphere

\subsection{The Central Motor Conduction Time (CMCT)}

A significant main effect of time was observed, Wilks' Lambda $=0.106, F(4,5)$ $=10.556, \mathrm{p}<0.012$.

During pairwise comparisons, investigating statistical differences within groups, significant differences between the CMCT at baseline and CMCT were visible on day 5 ( $\mathrm{p}=$ 0.006), between CMCT at baseline and CMCT on day $12(\mathrm{p}=0.014)$, between CMCT at baseline and CMCT on day $90(\mathrm{p}=0.029)$. Also, significant differences were observed between CMCT at day 5 and CMCT at day $12(\mathrm{p}=0.040)$ and between CMCT at day 30 and 
CMCT on day 90 ( $p=0.004)$. Non-significant differences were observed between any other possible pair of time points where the CMCT was measured. (Fig. 5)

Fig. 5 The effect of fampridine on the time obtained by the patients on the Central Motor Conduction Time (CMCT)

\subsection{The Central Motor Conduction Time (CMCT) from the right and left hemisphere in the lower limbs}

CMCT from the left hemisphere in the lower limbs showed a significant main effect of time (Fig. 6), Wilks' Lambda $=0.159, \mathrm{~F}(4,5)=6.612, \mathrm{p}=0.031$

Significant differences between the CMCT at baseline and CMCT were visible on day $12(\mathrm{p}=0.044)$ by the use of pairwise comparisons. Also, significant differences were observed between CMCT at day 30 and CMCT on day 90 ( $\mathrm{p}=0.011$ ). Non-significant differences regarding the $\mathrm{CMCT}$ in the lower limbs from the left hemisphere were observed between any other possible pair of time points where the CMCT was measured.

Regarding the Central Motor Conduction Time (CMCT) from the right hemisphere in the lower limbs a significant main effect of time was observed, Wilks' Lambda $=0.162, \mathrm{~F}(4,5)$ $=6.458, \mathrm{p}=0.033$.

When we examined the pairwise comparisons for statistical difference within groups related to the CMCT from the left hemisphere in the lower limbs, we observed significant differences only between CMCT at day 30 and CMCT on day $90(p=0.002)$.

Fig. 6 The effect of fampridine on the time obtained by the patients on the Central Motor Conduction Time (CMCT) from the left and right hemisphere in the lower limbs

\subsection{The Central Motor Conduction Time (CMCT) from the right and left hemisphere in the upper limbs}

Analysis of CMCT from the left hemisphere in the upper limbs revealed a significant main effect of time, Wilks' Lambda $=0.148, F(4,5)=7.221, p=0.026$. 
When we examined the pairwise comparisons for statistical difference within groups related to the CMCT from the left hemisphere in the upper limbs, we observed a statistically significant improvement between every time point in which this test was assessed (Fig. 7). Specifically, we found significant differences between CMCT at baseline and CMCT on day $5(\mathrm{p}=0.039)$, CMCT at baseline and CMCT on day $12(\mathrm{p}=0.003)$, CMCT at baseline and CMCT on day $30(p=0.001)$, CMCT at baseline and CMCT on day $90(p<0.001)$. Also, significant differences were observed between CMCT at day 5 and CMCT on day 12 ( $\mathrm{p}=$ 0.001), CMCT on day 5 and CMCT on day 12 ( $\mathrm{p}<0.001$ ), CMCT on day 5 and CMCT on day 90 ( $p<0.001)$. Furthermore, significant differences were observed between CMCT at day 12 and CMCT on day 30 ( $\mathrm{p}=0.005)$, CMCT on day 12 and CMCT on day 90 ( $\mathrm{p}=$ 0.001), CMCT on day 30 and CMCT on day $90(\mathrm{p}=0.002)$.

Regarding the Central Motor Conduction Time (CMCT) from the right hemisphere in the upper limbs a significant main effect of time was observed, Wilks' Lambda $=0.074, F(4,5)$ $=15.595, \mathrm{p}=0.005$.

Analysing in same manner the CMCT from the right hemisphere in the upper limbs, we observed a statistically significant improvement between every time point in which this test was assessed. Specifically, we found significant differences between CMCT at baseline and CMCT on day 5 ( $\mathrm{p}=0.001)$, CMCT at baseline and CMCT on day $12(\mathrm{p}<0.001)$, CMCT at baseline and CMCT on day 30 ( $\mathrm{p}<0.001)$, CMCT at baseline and CMCT on day 90 ( $\mathrm{p}<0.001)$. Also, significant differences were observed between CMCT at day 5 and CMCT on day 12 ( $p<0.001)$, CMCT on day 5 and CMCT on day 12 ( $p<0.001)$, CMCT on day 5 and CMCT on day 90 ( $\mathrm{p}<0.001)$. Furthermore, significant differences were observed between CMCT at day 12 and CMCT on day 30 ( $\mathrm{p}<0.001)$, CMCT on day 12 and CMCT on day $90(\mathrm{p}<0.001)$, CMCT on day 30 and CMCT on day $90(\mathrm{p}=0.034)$.

Fig. 7 The effect of fampridine on the time obtained by the patients on the Central Motor Conduction Time (CMCT) from the left and right hemisphere in the upper limbs

\section{Discussion}


According to our results, fampridine treatment significantly improves the quality of life from baseline to 90 days' time point $(p<0.001)$. Furthermore, our study agrees that a 12 days period after beginning the treatment is necessary to observe any significant improvement. There was no difference regarding the quality of life between baseline and day $5(\mathrm{p}=1)$, however, a significant positive difference was observed between the quality of life at baseline and quality of life after 12 days $(p<0.001)$, and also between quality of life after 5 days and quality of life after 12 days $(p<0.001)$. The statistically significant improvement continues after 30 days $(\mathrm{p}<0.001)$, but it stops after this time point, the difference between day 30 and day 90 are statistically non- significant $(p=1)$. To summarize, in our sample, statistically, fampridine treatment significantly improves the quality of life of the patients, after 12 days, yet this positive effect is diminishing and becomes non-significant after 30 days. In addition, our Wilks' Lambda analysis shows that there was a significant main effect of time on the quality of life $(p<0.001)$.

Somehow similar to the results obtained for the quality of life, the results for the 25foot walk test also showed improvement from baseline to the 90 days' time point $(\mathrm{p}<0.001)$. In addition, also a minimum of 12 days after the beginning of the fampridine treatment is required to observe statistically significant improvements. No significant differences were found between baseline and day $5(\mathrm{p}=0.132)$, but a significant difference was observed between day 5 and day 12 ( $\mathrm{p}<0.001)$, and also between baseline and day $12(\mathrm{p}<0.001)$. In contrast to the results from the quality of life, the improvement in the 25-foot walk test continued through the whole 90 days follow up of the study. In addition, our Wilks' Lambda analysis shows that there was a significant main effect of time on the result of the 25-foot walk test $(\mathrm{p}<0.001)$.

The results regarding the central motor conduction time (CMCT) also showed a significant main effect of time on the results obtained by the participants on this test $(\mathrm{p}<$ 0.001). In addition, a significant improvement was also observed between baseline and after 90 days of treatment $(p=0.029)$. Furthermore, improvements were also observed between baseline and day $5(\mathrm{p}=0.006)$, suggesting that it takes fampridine less time to have a positive effect on the CMCT compared to the 25-foot walk test and the quality of life. The observed improvements continued through day 12 of the treatment $(p=0.014)$. However, 
the positive effect of fampridine on the CMCT until the end of the 90 days of treatment (day 30-day 90, $\mathrm{p}=0.005)$.

The study has its limitations, as its weakness is especially related to the statistical significance while dealing with a small sample size. As a pilot study initially designed for at least 28 participants, it had an important dropout rate, as 13 of the initial patients stopped medication due to personal reasons within the first 5 to 12 days. It is to be mentioned that it was not related to side effects. Even in our small remaining lot, the decision of continuing the treatment beyond the first 2 weeks in patients without any important clinical amelioration shows interesting perspectives of both positive impacting the quality of life, as well as the use of TMS for monitoring the therapeutic response, facts we considered worth sharing.

As gait impairment continues to be one of the major problems in MS, involving high healthcare costs worldwide for both therapy attempts as well as scientific research. Fampridine, as a symptom controlling therapeutic agent for gait improvement among these patients has been showing positive effects throughout the last years, especially by association with physical therapy (13). In this context, we propose TMS as a useful tool not only for investigation of the relapse in MS, but also for follow-up of specific symptomatic treatments, such as Fampridine. It can be used for measurements of the motor evoked potential's amplitude (MEP), the central motor conduction time (CMCT) and the motor threshold. A decrease of CMCT and an increase of the MEP amplitude can early predict an improvement of speed, as correlated with the clinical evaluation on the T25-FW.

Fampridine is known to improve ambulation and endurance, but also, by the same mechanism of enhancing action potential formation in demyelinated axons by blocking potassium channels, it appears to improve cognitive fatigue as well $(14,15)$. Fampridine also seems to improve muscle strength and the rate of force development as measured not only by T25FW, but also by isokinetic dynamometry, Nine Hole Peg Test and other muscle strength or manual functions monitoring tests $(16,17)$. In other cases, there is mention of significant amelioration of the T25FW, whereas motor and cognitive fatigue seem to persist after 9 to 12 months (18). 
The QoL in MS patients can not only be affected by walking difficulties alone, but also by the related fatigue, which affects more than $80 \%$ of the patients. It leads to early retirement and is considered as the most burdensome clinical aspect of the disease by up to one out of four patients $(6,19)$. Fampridine, in this case, joins exercise therapy, therapeutic interventions on cognitive behaviour or symptomatic treatments for secondary sleep disorders against MS-related fatigue, as up to this moment, it seems to be the only therapeutic agent with promising results for treatment of both motor functions and motor fatigue (20). And due to its high tolerability profile, Fampridine is even likely to lead to favourable outcome of pregnancy (21).

It is not only the positive effect of Fampridine in improving walking that deserves to be mentioned, but also improvement of the psychological status (22-25). Either in double blind placebo-controlled studies or prospective monocentric open label trials, Fampridine has been proved to have effect upon patient self-reported psychological impact of MS, as well as a precognitive effect on verbal fluencies even in non-respondent to gait benefit patients, respectively (26). Therefore, by improving not only the physical-related issues in MS, but also cognitive fatigue and mood, Fampridine positively impacts QoL $(24,25)$. Thus, it significantly improves after Fampridine administration (27-29). Such already known facts contributed to our idea of including a very simple QoL quiz for our patients. The general state improved within our 4 patients that determined Fampridine continuation, finally leading to acceptable results.

In spite of the significant reduction on the T25FW, adverse events may occur during treatment with Fampridine (30, 31). These may include: dizziness, insomnia, falls, nausea and headaches. Ineffectiveness or inappropriate dosing count as well, and also anaphylactic reactions and seizures in rare cases. Adverse events might also include urinary tract infections (UTI), but there are studies that found similar results concerning the risk for UTI within dalfampridine patients and placebo groups $(31,32)$. Two of our patients experienced dizziness in the beginning of the study as the only adverse event, however, with amelioration within the first week.

Arguments for generally continuing the therapy are, for all that, enough. Especially because even in case of discontinuity of treatment, followed by a return to therapy, in spite 
of the decreased walking speed over time, there is still evidence of an improvement as compared to non-responders (33).

\section{Conclusions}

There are patients with no visible clinical improvement of speed on the T25-FW after 2 weeks on Fampridine. This might be related to the type of disease. Our study provides evidence that this situation could require a separate analysis of the results.

Mild electrophysiological modifications could be predictors for a future clinical improvement, if the therapy is continued, as correlated with QoL assessment.

In our study, the concept of false negative clinical response to Fampridine emerges, suggesting TMS as a useful tool for predicting even mild clinical benefits. We can therefore conclude that, at least for research purpose at this moment, early electrophysiological findings using TMS could be useful as a predictive tool for a future clinical amelioration.

\section{Declarations}

\section{Abbreviations}

CMCT: Central Motor Conduction Time

EDSS: Expanded Disability Status Scale

MS: Multiple Sclerosis

T25-FW: Timed-25-Foot-Walk test

TMS: Transcranial Magnetic Stimulation

- Ethics approval and consent to participate

The protocol was approved by the Ethics Committee of the University of Medicine and Farmacy “Gr. T. Popa”, Iași. 
- Consent for publication

Not applicable.

- Availability of data and material

The datasets used and/or analyzed during the current study are available from the corresponding author on reasonable request.

- Competing interests

The authors declare that they have no competing interests

- Funding

The current research was developed independently, with no funding involved.

- Authors' contributions

Trofin DM conceived the study, conducted the experiment, collected the data and took part in analysing the data and drafting the manuscript.

Trofin D contributed the data collection, led the data analysis and drafted the manuscript.

Prof. Bild W contributed to data analysis and manuscript drafting, critically appraised the manuscript and the revision.

Prof. Stamate T took part in drafting the manuscris.

Ciobica A participated in the data analysis process.

Ignat BE participated in data collecting and reviewed the manuscript.

All authors read and approved the final manuscript.

- Acknowledgements

Ignat BE has reviewed the manuscript and made valuable suggestions. Conflict of interest 
The authors declare no conflict of interest.

\section{References}

1. Arnold R, Huynh W, Kiernan MC, Krishnan AV. Ion Channel Modulation as a Therapeutic Approach in Multiple Sclerosis. Curr Med Chem. 2015;22(38):4366-78.

2. Huynh W, Pickering H, Howells J, Murray J, Cormack C, Lin CS, et al. Effect of fampridine on axonal excitability in multiple sclerosis. Clin Neurophysiol. 2016;127(7):2636-42.

3. Behm K, Morgan P. The effect of symptom-controlling medication on gait outcomes in people with multiple sclerosis: a systematic review. Disabil Rehabil. 2018;40(15):173344 .

4. Fragoso YD, Adoni T, Alves-Leon SV, Apostolos-Pereira SL, Barreira AA, Brooks JB, et al. Real-life experience with fampridine (Fampyra(R)) for patients with multiple sclerosis and gait disorders. NeuroRehabilitation. 2016;39(2):301-4.

5. Filli L, Zorner B, Kapitza S, Reuter K, Lorincz L, Weller D, et al. Monitoring long-term efficacy of fampridine in gait-impaired patients with multiple sclerosis. Neurology. 2017;88(9):832-41.

6. Ramio-Torrenta L, Alvarez-Cermeno JC, Arroyo R, Casanova-Estruch B, Fernandez O, Garcia-Merino JA, et al. A guide to treating gait impairment with prolonged-release fampridine (Fampyra $((\mathrm{R}))$ ) in patients with multiple sclerosis. Neurologia. 2018;33(5):327-37.

7. Lo AC, Ruiz JA, Koenig CM, Anderson BM, Olson KM, Triche EW. Effects of dalfampridine on multi-dimensional aspects of gait and dexterity in multiple sclerosis among timed walk responders and non-responders. J Neurol Sci. 2015;356(1-2):77-82.

8. Mejuto B, Castellano P, Castro C, Lopez LM. Assessment of the efficacy and safety of fampridine. Farm Hosp. 2017;41(2):283-91.

9. Institute for Quality and Efficiency in Health Care. Fampridine -- Benefit Assessment According to §35a Social Code Book V [Internet]. Cologne, Germany: Institute for 
Quality and Efficiency in Health Care (IQWiG); 2012 Apr Extract of Dossier Assessment No A12-06 IQWiG Dossier Assessment Extracts.

10. Alvarez-Payero M, Valeiras-Munoz C, Lion-Vazquez S, Pineiro-Corrales G, MunozGarcia D, Midaglia L. Experience with fampridine in clinical practice: analysis of a possible marker of clinical response. Int J Neurosci. 2017;127(10):915-22.

11. Pickering H, Murray J, Lin CS, Cormack C, Martin A, Kiernan MC, et al. Fampridine treatment and walking distance in multiple sclerosis: A randomised controlled trial. Clin Neurophysiol. 2017;128(1):93-9.

12. Brambilla L, Rossi Sebastiano D, Aquino D, Torri Clerici V, Brenna G, Moscatelli M, et al. Early effect of dalfampridine in patients with MS: A multi-instrumental approach to better investigate responsiveness. J Neurol Sci. 2016;368:402-7.

13. Ziemssen T, Prosser C, Haas JS, Lee A, Braun S, Landsman-Blumberg P, et al. Healthcare resource use and costs of multiple sclerosis patients in Germany before and during fampridine treatment. BMC Neurol. 2017;17(1):62.

14. Morrow SA, Rosehart H, Johnson AM. The effect of Fampridine-SR on cognitive fatigue in a randomized double-blind crossover trial in patients with MS. Mult Scler Relat Disord. 2017;11:4-9.

15. Jensen HB, Mamoei S, Ravnborg M, Dalgas U, Stenager E. Distribution-based estimates of minimum clinically important difference in cognition, arm function and lower body function after slow release-fampridine treatment of patients with multiple sclerosis. Mult Scler Relat Disord. 2016;7:58-60.

16. Jensen HB, Nielsen JL, Ravnborg M, Dalgas U, Aagaard P, Stenager E. Effect of slow release-Fampridine on muscle strength, rate of force development, functional capacity and cognitive function in an enriched population of MS patients. A randomized, double blind, placebo controlled study. Mult Scler Relat Disord. 2016;10:137-44.

17. Savin Z, Lejbkowicz I, Glass-Marmor L, Lavi I, Rosenblum S, Miller A. Effect of Fampridine-PR (prolonged released 4-aminopyridine) on the manual functions of patients with Multiple Sclerosis. J Neurol Sci. 2016;360:102-9. 
18. Ruck T, Bittner S, Simon OJ, Gobel K, Wiendl H, Schilling M, et al. Long-term effects of dalfampridine in patients with multiple sclerosis. J Neurol Sci. 2014;337(1-2):18-24.

19. Veauthier C, Hasselmann H, Gold SM, Paul F. The Berlin Treatment Algorithm: recommendations for tailored innovative therapeutic strategies for multiple sclerosisrelated fatigue. EPMA J. 2016;7:25.

20. Veauthier C, Paul F. [Therapy of fatigue in multiple sclerosis : A treatment algorithm]. Nervenarzt. 2016;87(12):1310-21.

21. Maillart E, Gout O, Lubetzki C, Carpentier B, Nizard J. Favorable outcome of a pregnancy after fampridine exposition during the first month. J Neurol Sci. 2016;370:158.

22. Gasperini C, Hupperts R, Lycke J, Short C, McNeill M, Zhong J, et al. Prolonged-release fampridine treatment improved subject-reported impact of multiple sclerosis: Item-level analysis of the MSIS-29. J Neurol Sci. 2016;370:123-31.

23. Macdonell R, Nagels G, Laplaud DA, Pozzilli C, de Jong B, Martins da Silva A, et al. Improved patient-reported health impact of multiple sclerosis: The ENABLE study of PR-fampridine. Mult Scler. 2016;22(7):944-54.

24. Pavsic K, Pelicon K, Ledinek AH, Sega S. Short-term impact of fampridine on motor and cognitive functions, mood and quality of life among multiple sclerosis patients. Clin Neurol Neurosurg. 2015;139:35-40.

25. Crayton H, Sidovar M, Wulf S, Guo A. Patient perspectives and experience with dalfampridine treatment in multiple sclerosis-related walking impairment: the step together program. Patient. 2015;8(3):283-91.

26. Magnin E, Sagawa Y, Jr., Chamard L, Berger E, Moulin T, Decavel P. Verbal Fluencies and Fampridine Treatment in Multiple Sclerosis. Eur Neurol. 2015;74(5-6):243-50.

27. Sagawa Y, Jr., Magnin E, Paillot L, Moulin T, Decavel P. Fampridine and quality of life in individuals with multiple sclerosis. Springerplus. 2016;5(1):1070.

28. Allart E, Benoit A, Blanchard-Dauphin A, Tiffreau V, Thevenon A, Zephir H, et al. Sustained-released fampridine in multiple sclerosis: effects on gait parameters, arm function, fatigue, and quality of life. J Neurol. 2015;262(8):1936-45. 
29. Lugaresi A. Pharmacology and clinical efficacy of dalfampridine for treating multiple sclerosis. Expert Opin Drug Metab Toxicol. 2015;11(2):295-306.

30. Costa-Arpin E, Pato A, Rodriguez-Regal A, Midaglia L, Yanez R, Munoz D, et al. Clinical response and tolerability of fampridine in clinical practice. Neurodegener Dis Manag. 2016;6(2):99-105.

31. Jara M, Aquilina T, Aupperle P, Rabinowicz AL. Safety profile of dalfampridine extended release in multiple sclerosis: 5-year postmarketing experience in the United States. Drug Healthc Patient Saf. 2015;7:169-74.

32. Kantor D, Chancellor MB, Snell CW, Henney HR, 3rd, Rabinowicz AL. Assessment of confirmed urinary tract infection in patients treated with dalfampridine for multiple sclerosis. Postgrad Med. 2015;127(2):218-22.

33. Goodman AD, Bethoux F, Brown TR, Schapiro RT, Cohen R, Marinucci LN, et al. Longterm safety and efficacy of dalfampridine for walking impairment in patients with multiple sclerosis: Results of open-label extensions of two Phase 3 clinical trials. Mult Scler. 2015;21(10):1322-31.

\section{Figures}




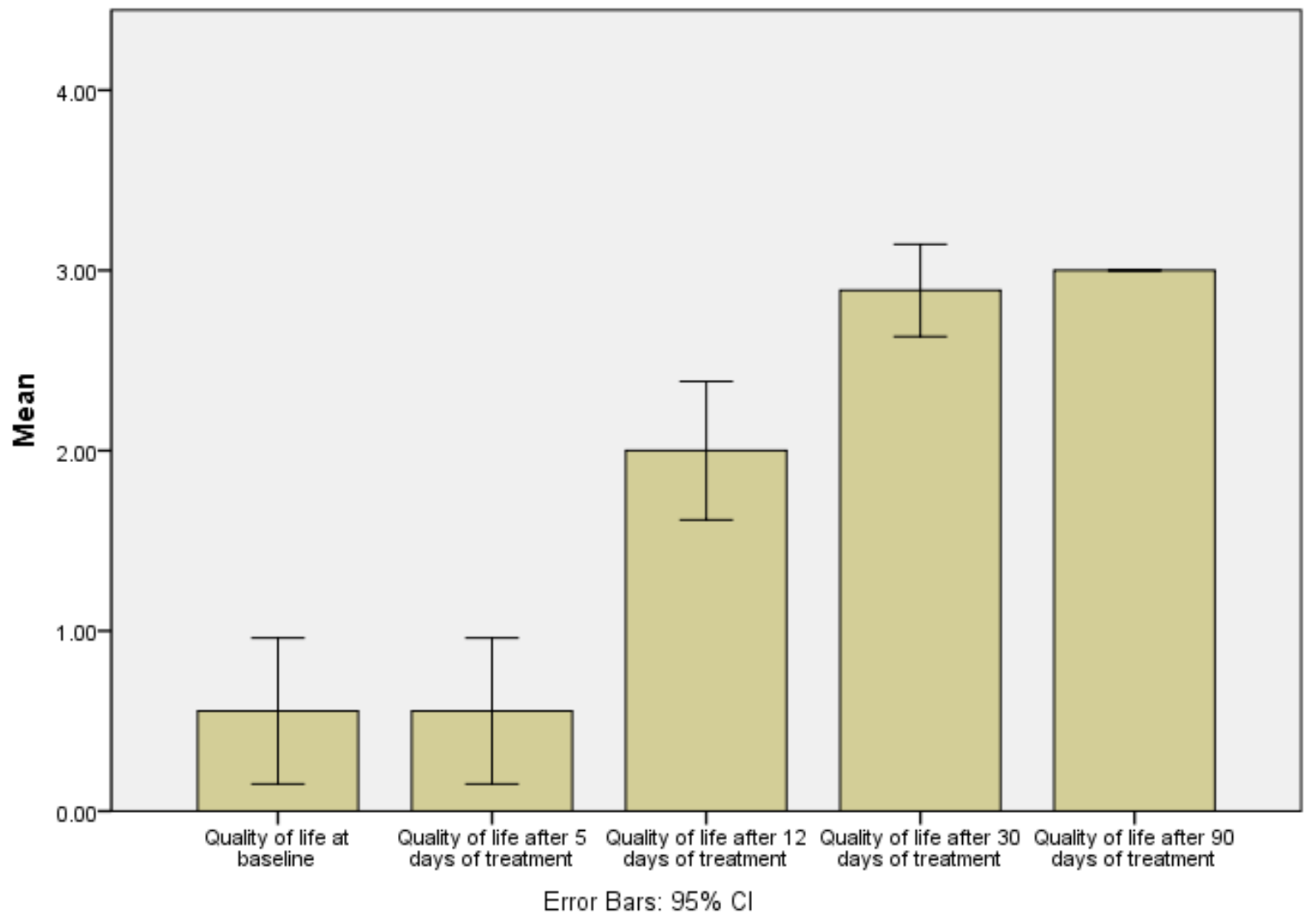

Figure 1

The effect of fampridine on the level of the quality of life 


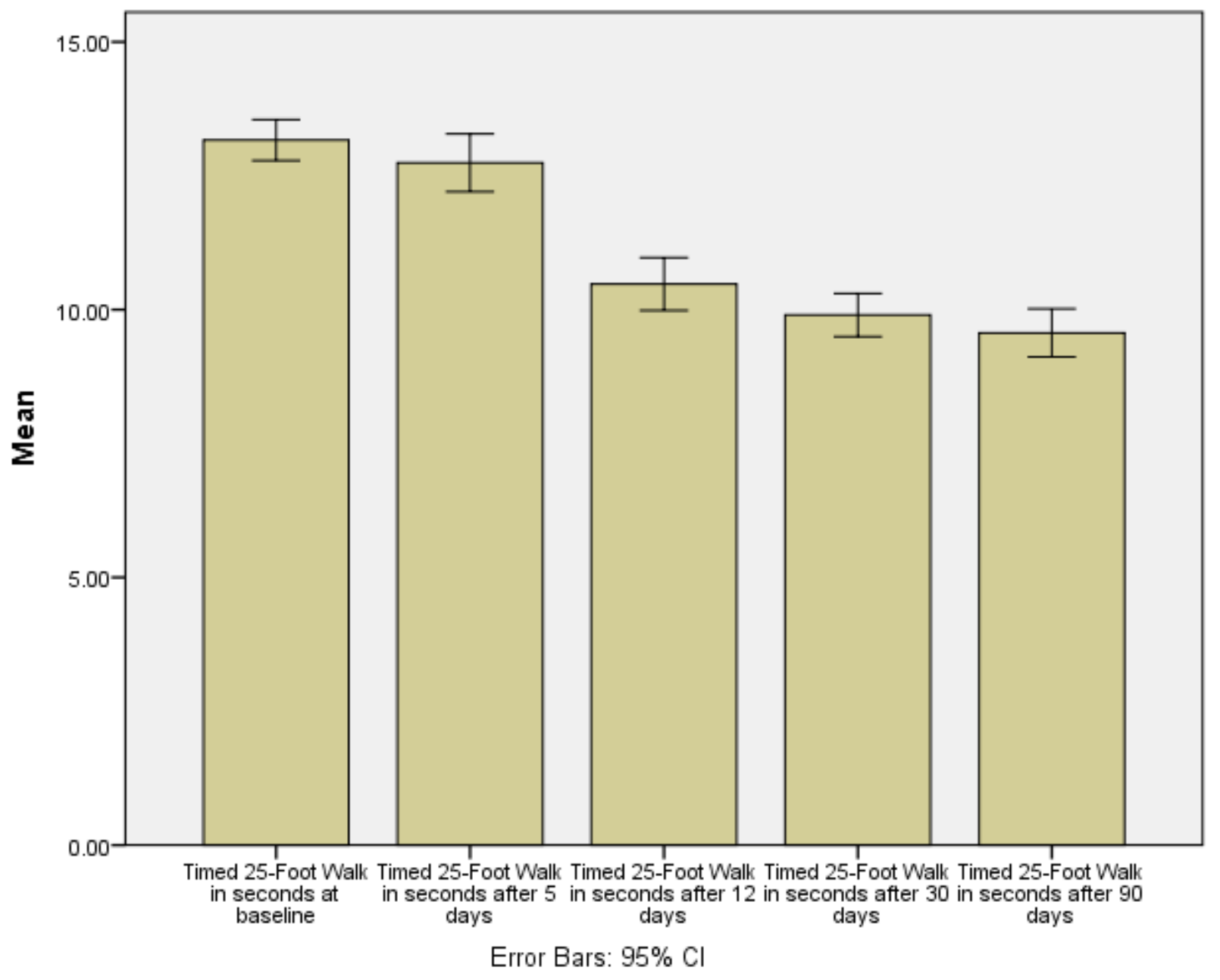

Figure 2

The effect of fampridine on the time obtained by the patients on the 25 Foot Walk test 


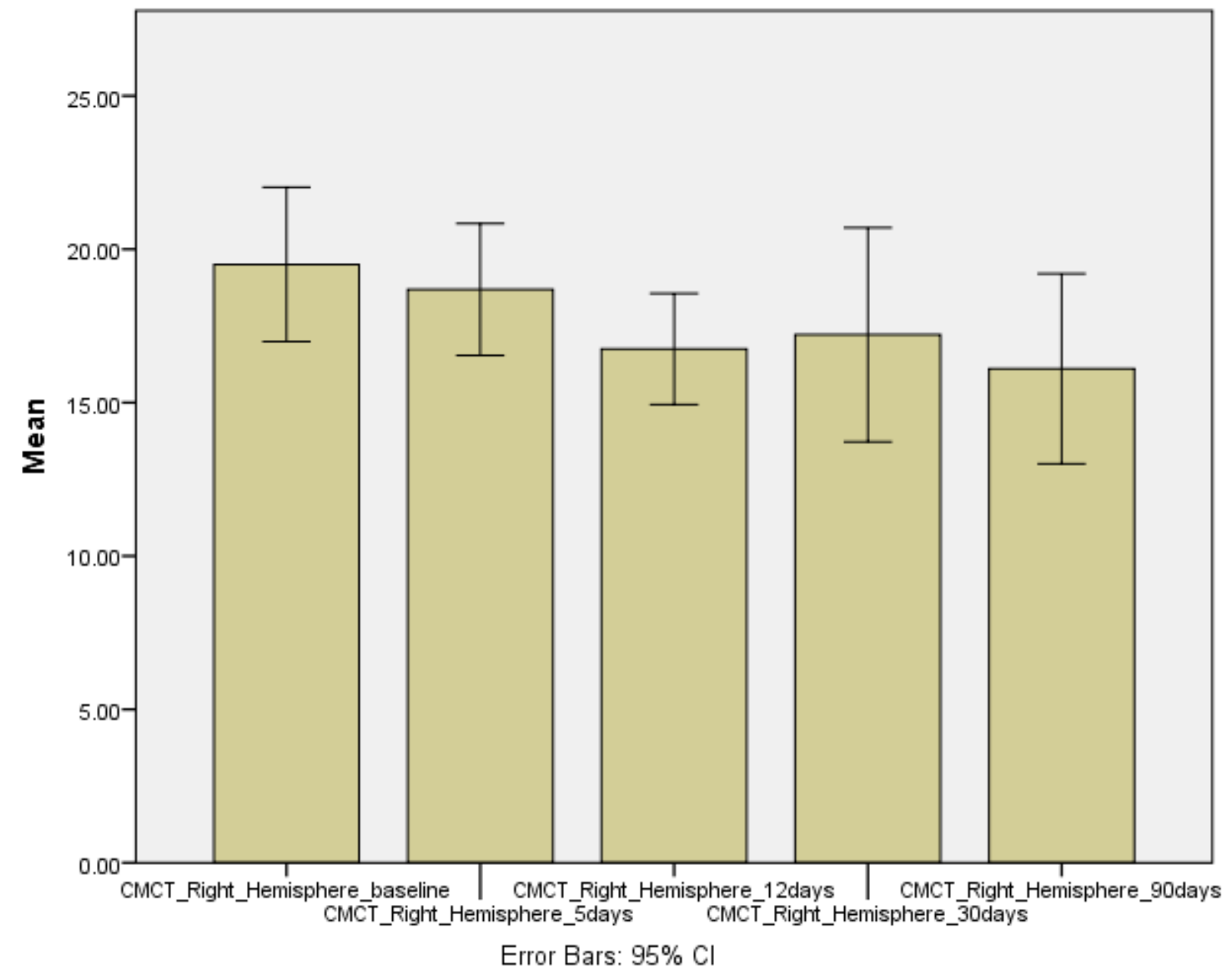

Figure 3

The effect of fampridine on the time obtained by the patients on the Central Motor Conduction Time (CMCT) from the right hemisphere 


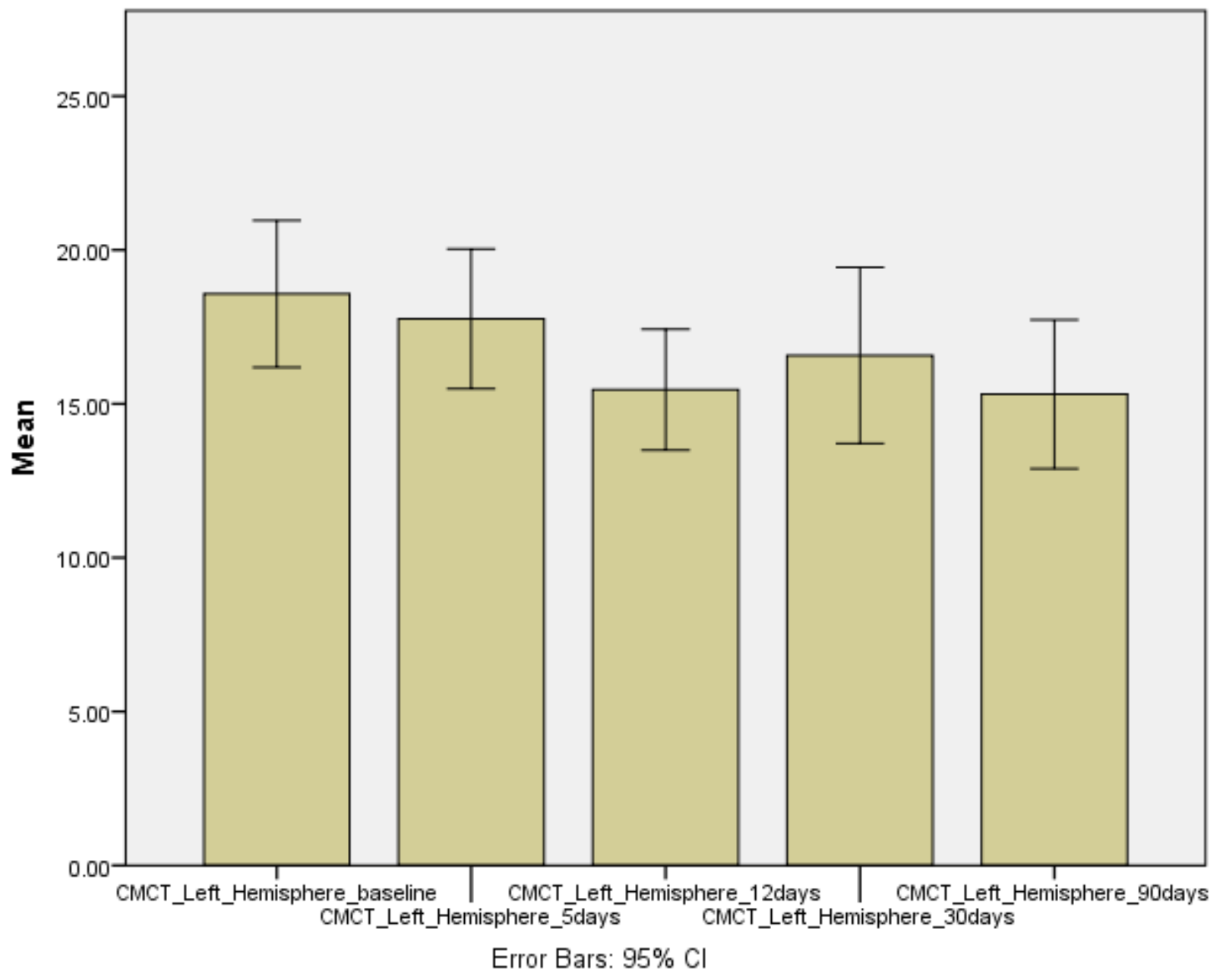

Figure 4

The effect of fampridine on the time obtained by the patients on the Central Motor Conduction Time (CMCT) from the left hemisphere 


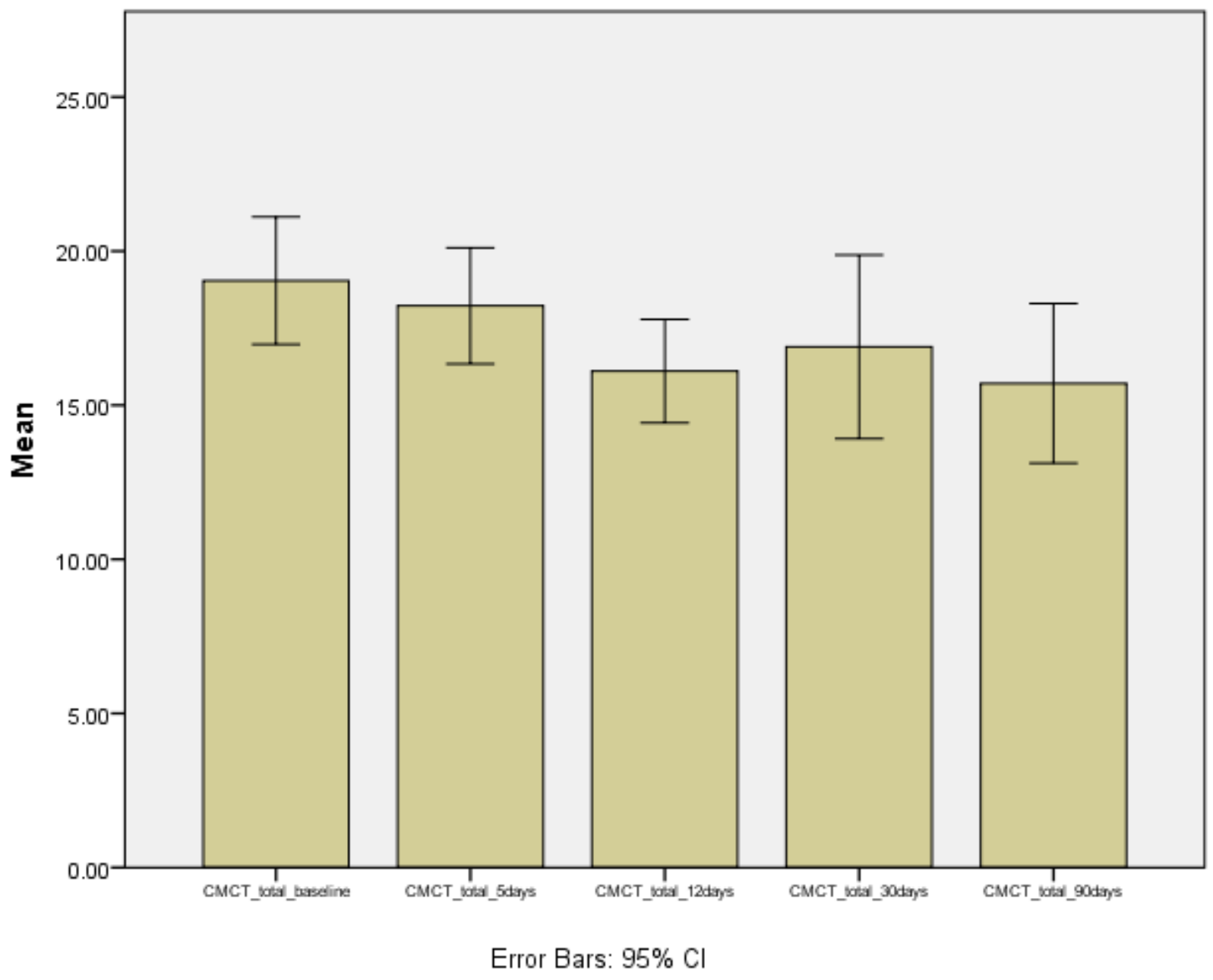

Figure 5

The effect of fampridine on the time obtained by the patients on the Central Motor Conduction Time (CMCT) 


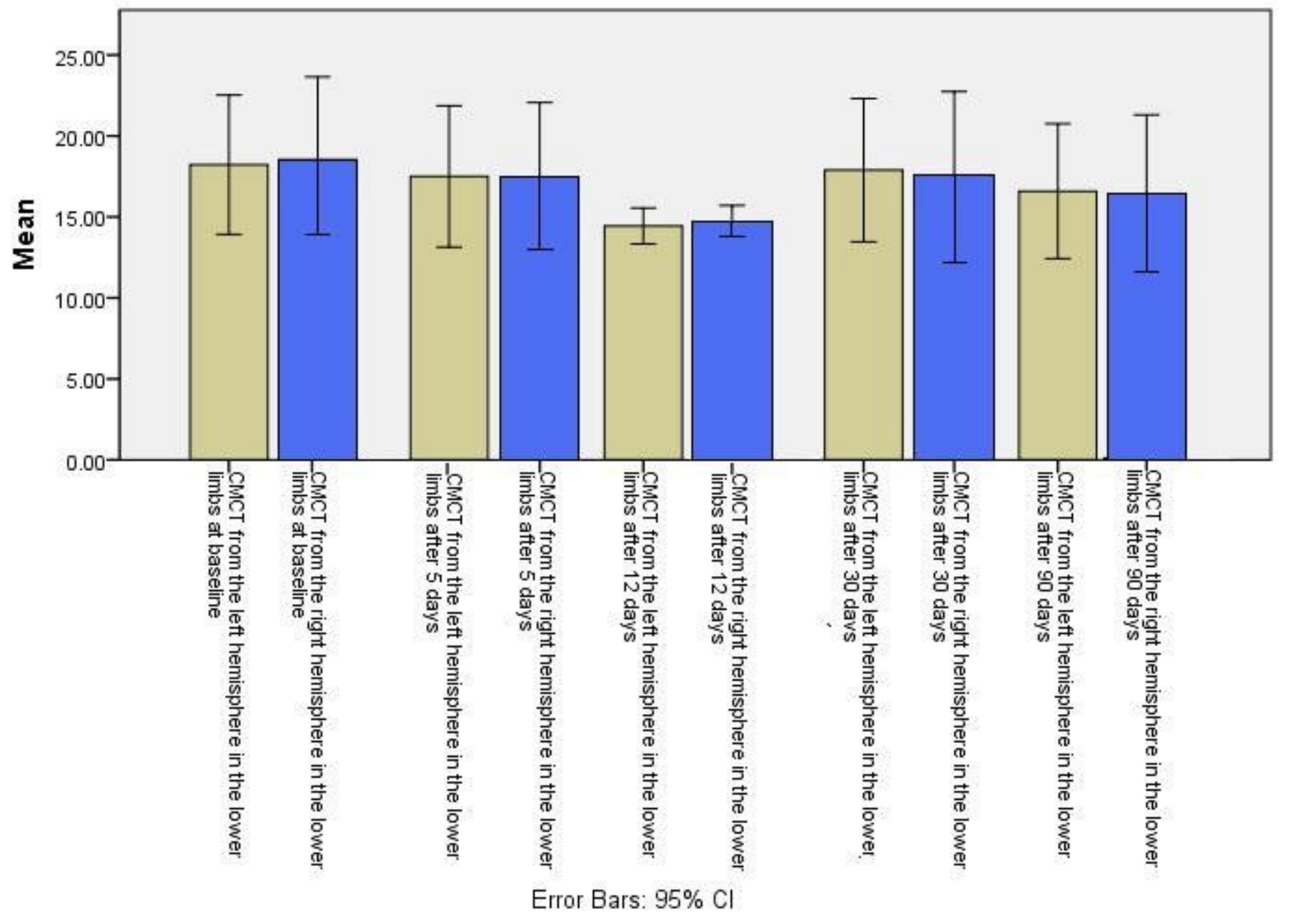

Figure 6

The effect of fampridine on the time obtained by the patients on the Central Motor Conduction Time (CMCT) from the left and right hemisphere in the lower limbs 


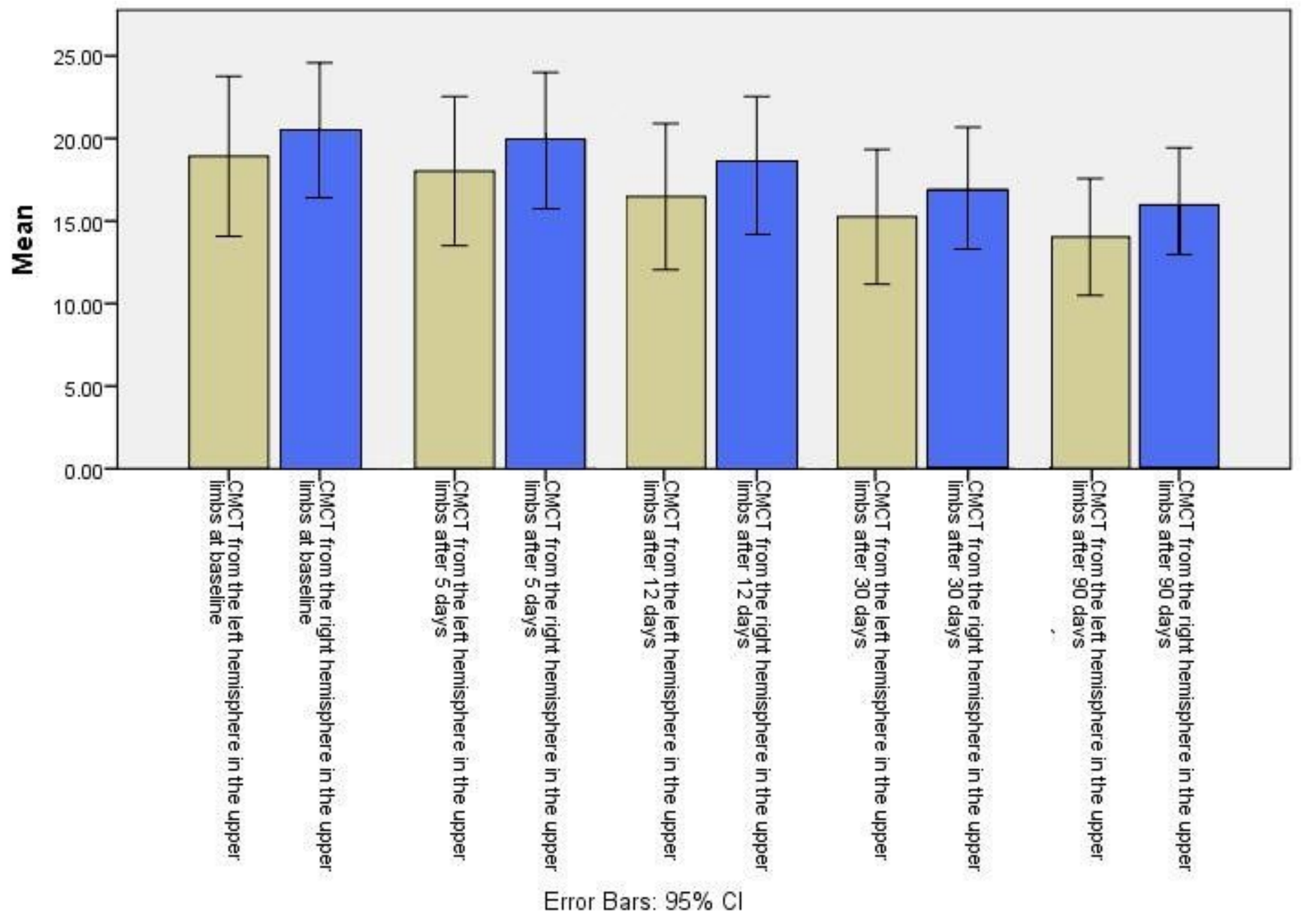

Figure 7

The effect of fampridine on the time obtained by the patients on the Central Motor Conduction Time (CMCT) from the left and right hemisphere in the upper limbs 